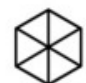 \\ LEUPHANA \\ UNIVERSITÄT LÜNEBURG
}

\section{Rhetorik der Kreativität}

Wuggenig, Ulf

Published in:

Kritische Kreativität

DOI:

10.14361/9783839446195-003

Publication date:

2019

Document Version

Verlags-PDF (auch: Version of Record)

Link to publication

Citation for pulished version (APA):

Wuggenig, U. (2019). Rhetorik der Kreativität: Über die internationale Zirkulation von Wörtern und Ideen. in K.

Kannler, V. Klug, K. Petzold, \& F. Schaaf (Hrsg.), Kritische Kreativität: Perspektiven auf Arbeit, Bildung, Lifestyle und Kunst (1. Aufl., S. 43-71). (Kulturen der Gesellschaft). transcript Verlag.

https://doi.org/10.14361/9783839446195-003

\section{General rights}

Copyright and moral rights for the publications made accessible in the public portal are retained by the authors and/or other copyright owners and it is a condition of accessing publications that users recognise and abide by the legal requirements associated with these rights.

- Users may download and print one copy of any publication from the public portal for the purpose of private study or research.

- You may not further distribute the material or use it for any profit-making activity or commercial gain

- You may freely distribute the URL identifying the publication in the public portal ?

Take down policy

If you believe that this document breaches copyright please contact us providing details, and we will remove access to the work immediately and investigate your claim. 


\section{Rhetorik der Kreativität}

\section{Über die internationale Zirkulation von Wörtern und Ideen}

Ulf Wuggenig

\section{Kreativitätskonzepte}

Es ist angesichts der Konjunktur des Wortes ,Kreativität‘ vielfach kaum bewusst, dass seine Einführung und sein Gebrauch im Deutschen historisch nicht sehr weit zurückgehen. Bevor dies und die internationale Diffusion dieses Wortes und entsprechender sprachlicher Äquivalente hier verdeutlicht werden, erscheint es naheliegend, ein paar Definitionen bzw. relevante Unterscheidungen in Zusammenhang mit diesem Konzept einzuführen, da sich der Begriff der Kreativität durch ein erhebliches Maß an semantischer Konfusion auszeichnet (vgl. Runco 2004; Cropley 2011; Sarsani 2011; Wuggenig 2016).

So wird Kreativität heute nicht selten einfach mit Neuerung und Innovation in Verbindung gebracht. Dafür steht im Englischen, aus dem das Wort im Sinne eines Imports in eingedeutschter Form übernommen wurde, etwa folgende Definition aus dem Cambridge Academic Content Dictionary 2017 sowohl in britischem als auch in US-amerikanischem Englisch: ,[T] $[$ he ability to produce original and unusual ideas, or to make something new or imaginative". Und dies ist, um ein Beispiel aus dem rezenten kulturwissenschaftlichen Diskurs zu zitieren, etwa auch der Fall in einer Monografie von John Hartley und Jason Potts (2014), in der nicht weniger versucht wird, als die britischen Cultural Studies und das, wofür dieses neogramscianische Paradigma steht, durch den Begriff der Cultural Science abzulösen. Bei dieser manifestartigen Schrift handelt es sich um eine Hybridisierung von Ideen insbesondere von Raymond Williams (1989 [1958]) („Culture is ordinary“) mit Juri Lotmans Semiotik und der Evolutionsökonomik, die sich an Joseph Schumpeter orientiert, dem „Propheten der Innovation“ (McCraw 2007). Hintergrund ist nicht zuletzt das Konzept der Creative Industries und die ökonomische Bedeutung, welche das auf diese Weise bezeichnete Wirtschaftssegment in der, wie es in jün- 
gerer Zeit zunehmend heißt, Creative Economy verspricht. Über die Manifeste von John Hartley und Jason Potts ist im Klappentext zu diesem Werk zu lesen: „Cultural Science introduces a new way of thinking about culture. Adopting an evolutionary and systems approach, the authors argue that culture is the population-wide source of newness and innovation; it faces the future, not the past" (2014, Herv.i.O.).

Gegenüber der Neigung, Kreativität primär oder ausschließlich mit der Schaffung oder der ,Schöpfung' des Neuen in Zusammenhang zu bringen - etwa auch in einem Kreativitätsdispositiv, das sich durch „drei historische Regime des Neuen“ auszeichne (Reckwitz 2016: 133) - stützt Niklas Luhmann den Begriff der Kreativität auf eine „Dreiheit“, nämlich die von „,neu / bedeutend / überraschend“ im Hinblick auf die „Zeit-, Sach- und Sozialdimension“ (Luhmann 1988: 13f.).

Nicht alles Neue oder Originelle kann nämlich beanspruchen, auch als interessant und wertvoll eingestuft zu werden, wie in ähnlicher Weise Margaret Boden aus philosophischer Perspektive betont. Da im Prinzip beständig die Schaffung von Neuem zu beobachten ist, hebt sie angesichts eines Überangebots an Neuerungen hervor, zudem noch zu berücksichtigen, ob dieses Neue auch als interessant und relevant erscheint. Sie schlägt deshalb vor, als „,kreativ“ ausschließlich „Ideen oder Artefakte“" zu bezeichnen, welche die Eigenschaften aufweisen, ,neu, interessant und wertvoll“" zu sein (Boden 2004: 1) - wobei die implizite Einschränkung zu beachten ist, dass Boden nicht, wie in anderer, neuerer Literatur zu finden, im Zusammenhang mit diesem Adjektiv auch soziale Einheiten wie Klassen, Städte oder Ökonomien vor Augen hat. Diese individualistische Verkürzung gilt auch für jene alternative Triade, welche Cropley (2011) in seiner Übersicht über Definitionen des Kreativitätsbegriffs in der Neuauflage der Encyclopedia of Creativity von Mark Runco und Steven Pritzker heranzieht, wenn es darin heißt, dass Kreativität die Schaffung von „etwas Neuem, Relevantem und Effektivem“ sei:

„Genuine creativity requires two further elements over and above mere novelty. A product or response must be relevant to the issue at stake and must offer some kind of genuine solution, that is, it must be effective. [...] Thus, creativity is nowadays widely defined as the production of relevant and effective novelty. What is meant by ,effective" may differ from, let us say, fine art to engineering to business.“ (Cropley 2011: 359, Herv.i.O.)

Margaret Boden unterscheidet aus kognitionswissenschaftlicher Perspektive in Form einer weiteren Triade drei hauptsächliche Formen der Kreativität. Eine erste Spielart bezeichnet sie als „kombinatorische Kreativität“, ausgezeichnet durch folgende Merkmale: „The first involves making unfamiliar combinations of familiar ideas. [...] These new combinations can be generated either deliberately or, often unconsciously“ (Boden 2004: 3; vgl. auch Sarsani 2011: 236). Für die zweite Form, 
die „explorative Kreativität“, führt sie zunächst den Begriff des „,konzeptuellen Raumes“ im Sinne eines strukturierten Denkstils ein, angeeignet bzw. eingelernt in einer Kultur, Subkultur, Peer Group oder anderen Art von sozialer Assoziation:

„Whatever the size of that space, someone who comes up with a new idea within that thinking style is being creative in the second, exploratory, sense. If the new idea is surprising not just in itself but as an example of an unexpected general type, so much the better. [...] All professional artists and scientists do this sort of thing.“ (Boden 2004: 4, Herv.i.O.)

Die dritte Form, die „transformative Kreativität“ impliziert eine weitreichendere Veränderung:

„The deepest cases of creativity involve someone's thinking something which, with respect to the conceptual spaces in their minds, they couldn't have thought before. The supposedly impossible idea can come about only if the creator changes the preexisting style in some way.“ (Ebd., 6, Herv.i.O.)

Neben den skizzierten Neigungen, gegenüber einem eindimensionalen Zugang zu Kreativität triadische Unterscheidungen zu treffen, findet sich vor allem in der psychologischen Literatur auch eine Reihe von Definitionsvorschlägen von führenden Kreativitätsforscher*innen, die sich lediglich an zwei Kriterien orientieren. Das ist der Fall, wenn beispielsweise Mark Runco von „kognitiven Prozessen“ spricht, „that lead to an original (e.g., novel, unique, or highly unusual) and adaptive (e.g., fitting, useful, or apt) insight, idea, or solution“ (1994: 11) oder Robert Sternberg und Todd Lubart Kreativität beschreiben als ,the ability to produce work that is both novel (i.e., original, unexpected) and appropriate (i.e., useful, adaptive concerning task constraints)“(1999: 3).

Der Schriftsteller und Wissenschaftler Arthur Koestler führte bereits in den 1960er Jahren in The Act of Creation (1964) den Begriff der „Bisociation“ für kreative Mechanismen der Kombination und Rekombination ein. Er brachte damit die „kombinatorische Kreativität“ im Sinne von Boden, die in assoziationistischen Theorien der Kreativität im Vordergrund steht, in einer später vielfach aufgegriffenen Weise auf den Begriff: „The creative act does not create something out of nothing $[\ldots]$; it combines, reshuffles, and relates already existing but hitherto separate ideas, facts, frames of perception, associative contexts. [...] I have proposed for it the term bisociation“ (Koestler 1964: 35).

Die vielleicht bekannteste Idee zur Bisoziation - die der ,kreativen Zerstörung ‘ - findet sich in der Wirtschaftstheorie. Sie geht in ihrer am stärksten verbreiteten Spielart auf Joseph Schumpeter zurück, dessen zunächst in Englisch formulierte These über den für den Kapitalismus charakteristischen „Sturm“ im Deutschen im 
Jahre 1950 noch unter Rekurs auf den Begriff des „Schöpferischen“ übersetzt wurde: „Dieser Prozess der ,schöpferischen Zerstörung“ ist das für den Kapitalismus wesentliche Faktum" (Schumpeter 1950: 137). Diese Diagnose des großen Gegenspielers von John M. Keynes und späteren Kulturhelden des Neoliberalismus wurde in der ökonomischen Theorie auch als ,Schumpeters gale“ bekannt. Der zur Zeit der Herstellung seiner Texte mit Bezügen zu Kreativität in den USA tätige österreichische Ökonom gebrauchte nämlich in Capitalism, Socialism, and Democracy, seiner auch mit soziologischen Ideen angereicherten Auseinandersetzung vor allem mit Marx, mehrfach die Formulierung ,the perennial gale of creative destruction“", um den aus seiner Sicht zentralen Mechanismus der kapitalistischen Dynamik zu umreißen (Schumpeter 2003: 84, 87f., 90). Schumpeter versuchte, den Innovationstendenzen im ökonomischen Feld Rechnung zu tragen, indem er auf die beständige ,industrial mutation“ im Rahmen des von ihm wie u.a. von Sombart und Weber (selten von Marx) vornehmlich als Kapitalismus bezeichneten Wirtschaftssystems verwies. Diese zeichne sich durch die folgende Dynamik aus: „[It] incessantly revolutionizes the economic structure from within, incessantly destroying the old one, incessantly creating a new one. This process of Creative Destruction is the essential fact about capitalism. It is what capitalism consists in and what every capitalist concern has got to live in“ (ebd., 83, Herv.i.O.).

Schumpeter, der die Neuerung speziell für das ökonomische Feld in einer vielzitierten Formulierung als „,neue Kombination von Produktionsfaktoren“ fasste, ${ }^{1}$ unterschied in einer späteren Arbeit, die das Adjektiv „creative“ sogar im Titel trägt (The Creative Response in Economic History, 1947), zwischen „,adaptiven“ und „kreativen Reaktionen“ einer Ökonomie oder eines ihrer Sektoren. Zur Kennzeichnung solcher Reaktionen orientiert sich Schumpeter an einer einfachen Idee von Neuerung, wenn er über die kreative Reaktion schreibt, dass von einer solchen unter folgender Bedingung gesprochen werden könne: ,[W]henever the economy or an industry or some firms in an industry do something else, something that is outside of the range of existing practice, we may speak of creative response" (Schumpeter 1947: 150). Kreativen Reaktionen im ökonomischen Feld schreibt Schumpeter zu, sich durch zumindest drei wesentliche Merkmale auszuzeichnen:

1 Schumpeters Innovationsbegriff, der auf die Rekombination vorhandener Elemente abhebt, wurde vielfach auf andere Bereiche übertragen und verallgemeinert, so nicht zuletzt auf die „soziale Innovation“, über die Josef Hochgerner unter Rekurs auf „creation“ schreibt: ,[S]ocial innovation can be defined as a new combination of social practices. In order to qualify as social innovations, such combinations or the creation and implementation of absolutely new practices must be intentional, aiming at solving a social issue, and produce effects in terms of novel social facts. Implementation and impact distinguish social innovations from social ideas“ (Hochgerner 2012: 87). 
1. Sie können nur ex post verstanden werden, praktisch nie ex ante, und lassen sich somit nicht induktiv aus bekannten Fakten über Inferenzschlüsse vorhersagen.

2. Kreative Reaktionen formen den gesamten Verlauf nachfolgender Ereignisse und ihrer längerfristigen Effekte. Auch in diesem Zusammenhang betont er in nicht zu übersehender Abgrenzung zu (geschichts-)teleologischen Vorstellungen deren Indeterminiertheit:

„Creative response changes social and economic situations for good, or, to put it differently, it creates situations from which there is no bridge to those situations that might have emerged in its absence. This is why creative response is an essential element in the historical process; no deterministic credo avails against this.“ (Ebd., 150)

3. Im Hinblick auf die individuelle Ebene heißt es schließlich in Formulierungen, die implizit auch gegen die Vorstellung, dass jede*r kreativ sein könne, gerichtet sind:

„Thirdly, creative response - the frequency of its occurrence in a group, its intensity and success or failure - has obviously something, be that much or little, to do

(a) with quality of the personnel available in a society,

(b) with relative quality of personnel, that is, with quality available to a particular field of activity relative to quality available, at the same time, to others, and

(c) with individual decisions, actions, and patterns of behavior." (Ebd.)

Hieraus folgt für Schumpeter auch, dass die kreative Reaktion im ökonomischen Feld mit der unternehmerischen Funktion assoziiert ist, sodass Kreativität, vermittelt über die Feder eines der maßgeblichen Ökonom*innen des letzten Jahrhunderts, zu einem der wesentlichen Aspekte der ,enterprise culture“ im Sinne der individualistischen Kultur von Mary Douglas (1996) und ihrer rhetorischen Propaganda und Rechtfertigung wird: „Accordingly, a study of creative response in business becomes coterminous with a study of entrepreneurship“" (Schumpeter 1947: 150).

Dabei ist die kreative Reaktion bei Schumpeter ebenso wie die unternehmerische Funktion und die Figur des Unternehmers auf das engste mit jener Idee der Neuerung verbunden, die sich auf kombinatorische Kreativität im Sinne von Boden stützt. Über den Unternehmer, diesen ,,agent of innovation“, ist zu lesen:

„Seen in this light, the entrepreneur and his function are not difficult to conceptualize: the defining characteristic is simply the doing of new things or the doing of things that are already being done in a new way (innovation). [...] It should be observed at once that the ,new thing' need not be spectacular or of historic importance.“ (Ebd., 151) 
Schumpeters auf den Kapitalismus angewandte Spielart der Idee der Bisoziation ist durchaus älteren Ursprungs. Sie lässt sich bereits bei Friedrich Nietzsche, Karl Marx und Michail Bakunin nachlesen. Unter den bürgerlichen Ökonomen war es der schillernde Werner Sombart, der lange vor Schumpeter schrieb: „Wiederum aber steigt aus der Zerstörung neuer Geist hervor“ (2013 [1913]: 207). Dieser dann von Schumpeter populär gemachte Gedanke hat letztlich jedoch, worauf Hugo und Erik Reinert (2006) verweisen, nicht-westliche Ursprünge: Zu denken ist etwa an die mit dem hinduistischen Gott Shiva verbundenen Vorstellungen der Verbindung von Gegensätzen oder auch an den Yoruba-Gott Ogun, auf den sich Wole Soyinka in seiner Nobelpreisrede des Jahres 1986 bezieht: „Meine kreative Muse ist Ogun, der Gott der Kreativität und Zerstörung“ (Soyinka 1986).

Koestler, zu dem wir nun zurückkehren, verdichtet die Idee der Bisoziation in ihrer allgemeinen Form visuell zu dem folgenden dynamischen Modell, in welchem er die Verschränkung zweier autonomer bzw. divergierender Bezugsrahmen (M1 und M2) mit je unterschiedlichen Eigenschaften (,wavelengths“) als kreative Idee oder Situation konzeptualisiert: In dieser Idee oder Situation (L), vergleichbar einem Schnittpunkt zweier Ebenen im Raum, werden die vormals getrennten Matrizen miteinander verbunden. Dadurch speist sich L aus zwei Kontexten und ist nicht nur mit einem Bezugsrahmen assoziiert, sondern mit zweien bisoziiert (vgl. Koestler 1964: 35).

Abbildung 1: Die Bisoziation von zwei Ebenen bzw. Bezugsrahmen, 1964

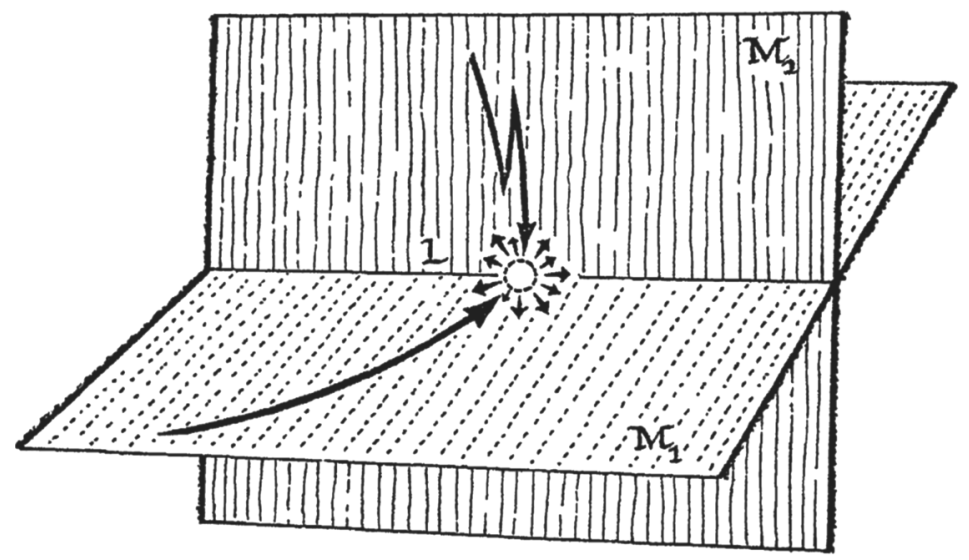

Quelle: Arthur Koestler, The Act of Creation, S. 35 
Koestler erläutert zu diesem Modell:

„I have coined the term ,bisociation“ in order to make a distinction between the routine skills of thinking on a single ,plane', as it were and the creative act, which, as I shall try to show, always operates on more than one plane. The former may be called single-minded, the latter a double-minded, transitory state of unstable equilibrium where the balance of both emotion and thought is disturbed." (Ebd.) $)^{2}$

Diese Idee wird heute in den Kognitionswissenschaften in der Theorie der kognitiven Vermischung (cognitive blending) weitergeführt. Fauconnier und Turner beziehen sich in der Formulierung ihres Forschungsprogramms auch auf den Begriff der „,ognitive fluidity“ (2002: 37ff.) des Archäologen Stephen Mithen, der sowohl an Koestler als auch an Boden anknüpft und in der Entwicklung dieser Art von kognitiver Flexibilität einen ,,big bang“ in der menschlichen Evolution sieht:

„Margaret Boden has argued that creative thought can be explained ,in terms of the mapping, exploration, and transformation of structured conceptual spaces'. Her definition of conceptual spaces is vague; she describes them as a style of thinking-in music, sculpture, choreography, chemistry, etc. 'In spite of this vagueness, the idea of transforming conceptual spaces is intuitively appealing. It has a close association with the earlier notions of Koestler, who has described creative thinking as arising from the sudden interlocking of two previously unrelated skills or matrices of thought, and the contemporary ideas of Perkins, who uses the terminology of ,klondike spaces' and argues that these are often systematically explored in the process of creative thinking. In this regard, while creative thinking is clearly part of ordinary thinking, and not something restricted to ,geniuses', we can nevertheless see the potential for how particularly creative thought may arise from quite unusual transformations of conceptual spaces undertaken by particular individuals in particular circumstances.“ (Mithen 1998: 123)

Bei der zeitgenössischen Weiterentwicklung der Idee der Bisoziation wird die Zusammenführung nicht bloß zweier getrennter oder entgegengesetzter Rahmungen modelliert, wie z.B. Schöpfung und Zerstörung oder Kunst und Wirtschaft, sondern auch die von komplexeren, multiplen Verknüpfungen von Ideen, Wahrnehmungsrahmen, nicht aufeinander bezogener „Matrizen des Denkens“ (Koestler 1964: 38) und ,verschiedener Domänen“ (Fauconnier/Turner 2002: 15).

Im Gegensatz zur Psychologie war in den Sozialwissenschaften jenseits der Wirtschaftstheorie die Beschäftigung mit Kreativität, wie Luhmann noch in den 1980er Jahren unterstrich, lange unterblieben. In jüngerer Zeit kam es jedoch nicht

2 Ein Beispiel für ein an Koestler anschließendes Kreativitätskonzept aus dem italienischen Kontext entfaltet der Beitrag von Gernot Waldner in diesem Band. 
nur zu einer sozio-kulturellen Wende in der psychologisch geprägten Kreativitätsforschung (vgl. Glaveanu et al. 2019), sondern es wandten sich in den beiden letzten Jahrzehnten auch Sozialwissenschaftler*innen, insbesondere aus Soziologie und Ökonomik, dem Thema vermehrt zu. Diese Tendenzen werden verdeutlicht durch Begriffsbildungen wie „Creative Class“ (Florida 2002), „Creative Industries“ (Caves 2000), „Creative City“ (Landry 2000), „Creative Economy“ (Howkins 2001), „Creative Capital“ (Kratke 2011), „Creative Economics“ (Menger 2014), „Kreativitätsdispositiv“ (Reckwitz 2012) oder - in kritischer Wendung - ,der neue“ bzw. „dritte Geist des Kapitalismus“. Letzterer bzw. die Phase sozio-ökonomischer Entwicklung, in der er sich herausgebildet hat, ist, wie Luc Boltanski und Ėve Chiapello, die Urheber*innen dieses Konzeptes, formulierten, stichwortartig u.a. durch ,permanenten Wandel“, ,unscharfe Organisation“ und nicht zuletzt „Kreativität und Innovation“ (2002: 7) zu charakterisieren. Kreativität in der von ihnen beschriebenen netzwerk- und projektbasierten Welt des zeitgenössischen Kapitalismus kann weder als eine Schöpfung ex nihilo verstanden werden noch als eine von isolierten Individuen, wie in historisch früheren Phasen. Sie erscheint nun im Rahmen der ,projektorientierten Cité" vielmehr als verteilt in ihrer Form und eingebettet in soziale Verbindungen:

„The projective city shares with the inspirational city the importance assigned to creativity and innovation (as attested, for example, by the use made of the paradigm of the network in the dominant currents of the sociology of innovation). Similarly, both these cities stress the uniqueness of beings and things, whose very difference creates value (not their ability to merge into collective forms, as is the case, for example, in the industrial city). But these similarities are superficial, and even misleading. In effect, whereas in the inspirational city people are creative when they are separated from others, withdrawn into themselves as it were, into their internal being - the only place where they can enter into direct relation with a transcendent source of inspiration (the supernatural) - or buried in the depths of the psyche (the unconscious), in the projective city creativity is a function of the number and quality of links. Moreover, it is a matter of recombination, rather than creation ex nihilo, and readily assumes a ,distributed' form (as one talks of ,distributed intelligence'), with responsibility for innovation being allocated between different actors.“ (Boltanski/Chiapello 2018: 173)

Denkt man an weitere wichtige Unterscheidungen im Zusammenhang mit Kreativität, so sind mit Boden (2004) auf der Ebene individueller Akteur*innen „Historical Creativity“ (kurz „H-Creativity“), die sich auf etwas bezieht, das geschaffen oder entdeckt wurde, was niemand zuvor geschaffen oder entdeckt hat, und „Psychological Creativity“ („P-Creativity“) auseinanderzuhalten, welche sie wie folgt beschreibt: „P-Creativity involves coming up with a surprising, valuable idea that's new to the person, who comes up with it. [...] But if a new idea is H-creative, that 
means that (so far as we know) no one else has had it before: it has arisen the first time in human history“ (Boden 2004: 2; vgl. auch Sarsani 2011: 231). Es ist wohl kaum nötig zu betonen, dass in Domänen wie Kunst, Wissenschaft und Technologie vor allem $\mathrm{H}$-Kreativität von Interesse ist.

In ähnlicher Weise schlägt Keith Simonton (2013) vor, zwischen Kreativität alltäglicher Art und Kreativität, die mit der Umgestaltung von ganzen Domänen verbunden ist und in das kulturelle Gedächtnis eingeht, zu differenzieren. Auf Letztere hebt auch die Sozialpsychologin Teresa Amabile ab, die sich einen Namen im Feld der Kreativitätsforschung gemacht hat:

„Kreativität ist jede Handlung, Idee oder Sache, die eine bestehende Domäne verändert oder eine bestehende Domäne in eine neue verwandelt. Und ein kreativer Mensch ist eine Person, deren Denken oder Handeln eine Domäne verändert oder eine neue Domäne begründet. Dabei darf man aber nicht vergessen, dass eine Domäne nur durch die explizite oder implizite Zustimmung des dafür verantwortlichen Feldes verändert werden kann.“ (Amabile 1996: 33)

Die Form von Kreativität, welche in der Lage ist, Paradigmenwechsel im Sinne von Thomas S. Kuhn (1970) nach sich zu ziehen oder auch ,symbolische Revolutionen“ im Sinne von Pierre Bourdieu (2013: 13), welche kognitive Strukturen umfassend verändern, wird in der Literatur oftmals - aber nicht notwendigerweise mit Genievorstellungen verbunden.

Katherine Giuffre (2009) hebt die Differenzen zwischen individualistischen und holistischen Zugängen hervor. Erstere verstehen Kreativität in erster Linie als ein Attribut von Individuen, Letztere als ein Produkt von sozialen Kräften, Strukturen, Milieus oder der Einnahme bestimmter sozialer Positionen und deren Inkorporierung im Habitus, also in Strukturen von Wahrnehmung und Klassifikation, sowie in generativen Prinzipien des Handelns von Akteur*innen. In welcher Weise Kreativität als sozialer Prozess auch als ein netzwerkbasierter verstanden wird, lässt sich etwa am Beispiel der Studie von Randall Collins (1987) zeigen: Kreativ zu sein bedeutet aus einer solchen Sicht nichts anderes, als in der Lage zu sein, Techniken einzusetzen, mit deren Hilfe etwas berühmt gemacht werden kann. Kreativität stellt sich zwar über Individuen her, aber Erfolg schafft man nicht alleine. Kreativität hat mit distinktiven Techniken und mit Nischen in Welten zu tun, die sich aus Rivalen und Rivalinnen, einem Publikum und Gefolgsleuten zusammensetzen. ${ }^{3}$

3 DiMaggio (2011: 289ff.) bietet darüber hinaus einen Überblick über soziologische Studien zu Kreativität in kulturellen Netzwerken. Dies mag man mit einem Überblick über die psychologische Kreativitätsforschung, die solche Zugänge nicht im Blick hat, vergleichen (vgl. 
Eine weitere Spielart nicht-individualistischen Denkens über Kreativität, die sich teilweise auch in der neueren Kreativitätspsychologie findet, ist das Verständnis von Kreativität als einer sozialen Zuschreibung bzw. als eines Labeling-Prozesses (vgl. Becker 2017). So lautet eine Hauptthese in einer der bekanntesten psychologischen Arbeiten zu Kreativität: ,[N]othing is, or is not, creative in and of itself. Creativity is [a] judgment“ (Gardner 2011: 36). Die Bedeutung der ,soziokulturellen Validierung“" von Kreativität wird in ähnlicher Weise wie von Teresa Amabile (1996) auch von Mihaly Csikszentmihalyi (2010: 48, 2014) betont, der in einem systemtheoretischen Bezugsrahmen die Notwendigkeit der sozialen Anerkennung von H-Kreativität und die Bedeutung von Türsteher*innen in einem sozialen Feld, welches Neuerungen nach feldtypischen Relevanzkriterien bewertet und auswählt, hervorhebt.

\section{Creative-Industries-Politik}

Eines der in der Folge wirkungsmächtigsten Beispiele für ein von soziologischen, aber auch neueren psychologischen Zugängen deutlich abweichendes, in starkem Maße individualistisches Verständnis von Kreativität lässt sich zugleich für einen Exkurs in die Felder von Kultur- und Wirtschaftspolitik nutzen. Eine explizit individualistische Vorstellung von Kreativität findet sich nämlich in einem jener Dokumente, die in den 1990er Jahren, ausgehend von Großbritannien, einen politischen Kreativitätshype in etlichen Ländern der EU, aber auch darüber hinaus, ausgelöst haben.

Angesprochen ist damit jene rückblickend mittlerweile als ,Creative-Industries-Politik‘ bezeichnete Strömung der 1990er Jahre, für die das Dokument The Creative Nation der australischen Labour Party von 1994 ein wesentlicher Ausgangspunkt war (vgl. Rowe et al. 2016). Auf diesen Ausgangspunkt in Australien folgte im Jahr 1997 die Einrichtung der Creative Industries Taskforce durch das neue ministeriale Department for Media, Culture and Sport (DMCS) in London, welches im Zuge des Aufstiegs von New Labour unter Tony Blair geschaffen wurde. 1998 lancierte dieses Ministerium das „Creative Industries Mapping Document“. Darin findet sich eine Definition von Creative Industries (CI), die sich ausdrücklich auf einen individualistischen Begriff von Kreativität stützt. Der vielzitierte Satz in diesem Dokument, der den vormals im britischen Diskurs vorherrschenden Begriff der Cultural Industries auf realpolitischer Ebene ablösen sollte, definiert Creative Industries als ,those industries which have their origin in indivi-

Runco 2004). Zu soziologischen Zugängen zur Kreativität generell vgl. Sawyer (2011) und Burns/Machado/Corte (2015a, b) sowie die älteren Arbeiten von Joas (1996) und Popitz (2000) und die neuere Studie von Reckwitz (2012) aus dem deutschsprachigen Raum. 
dual creativity, skill and talent and which have a potential for wealth and job creation through the generation and exploitation of intellectual property“ (DCMS 1998: 4).

Der Hintergrund zu diesem Versuch, den Kreativitätsbegriff zu instrumentalisieren und populär zu machen, ist dabei sehr aufschlussreich: Der Nettoinlandsproduktanteil der insgesamt 13 in dem Dokument als kreativ klassifizierten Industrien, deren Auflistung mit Werbung und Architektur beginnt und mit Software und Rundfunk endet, betrug zu dieser Zeit nicht weniger als fünf Prozent. Dies ist einer der wesentlichen Gründe, warum diesen Sektoren ein zunehmendes Maß an Aufmerksamkeit von Ökonom*innen und Politiker*innen gewidmet wurde. Eine zentrale Hypothese einer Studie von estnischen Wissenschaftler*innen, welche die auf den Kreativitätsbegriff gestützten politischen Strategien seit den 1990er Jahren untersuchten, ist diffusionstheoretischer Art: Mit ihr wird postuliert, dass die in der Literatur als ,Creative-Industries-Politik' bezeichnete Strategie sich in den 1990er Jahren ausgehend von Großbritannien in verschiedene Länder verbreitete und eine Homogenisierung sowohl der Terminologie im Bereich von Kulturund Wirtschaftspolitik als auch von institutionellen Strukturen herbeiführte. Genauer untersucht wurde von den Autor*innen die Creative-Industries-Politik für Amsterdam, Barcelona, Berlin, Birmingham, Helsinki, Oslo, Riga, Stockholm, Tallinn, Vilnius und Warschau (vgl. Tafel-Viia et al. 2014). Die These über einen wesentlichen ersten Mechanismus der CI-Politik, den von Großbritannien ausgehenden „Politiktransfer über die Welt“ (Tafel-Viia/Lassur 2015: 19), die sie für die untersuchten Städte bzw. Länder bestätigt fanden, erstreckte sich darüber hinaus auf asiatische Länder wie China und Malaysia. Die Autor*innen kennen auch noch einen zweiten Mechanismus, nämlich den der „Pfadabhängigkeit“ im Sinne relativ autonomer Formen der CI-Zielverfolgung in Abhängigkeit von Stadt, Region und Land (ebd., 21). Die Annahme eines homogenen Transfers ist jedoch gerade am Beispiel der deutschsprachigen Länder zu relativieren, da, wie noch zu zeigen sein wird, die Pfadabhängigkeit in diesem Fall relativ stark ausgeprägt war.

Auf etwas andere Weise, nämlich auf der Grundlage von Inhaltsanalysen einschlägiger Forschungsliteratur, haben die belgischen Sozialwissenschaftlerinnen Katja Segers und Ellen Huijgh (2006) diese Wende zur homogenisierenden Creative-Industries-Politik eingehend untersucht. Die folgenden Ausführungen stützen sich auf diese Analyse.

Segers und Huijgh unterscheiden eine erste Phase von internationaler Kulturpolitik nach dem Zweiten Weltkrieg bis 1974, die vom Konzept der ,Kulturindustrie‘ geprägt war, eine zweite von 1975 bis 1995, die vom Begriff der ,Kulturindustrien“ im Plural bestimmt war, und eine dritte ab 1995, in welcher der Begriff der ,Creative Industries' dominiert.

Ein wesentlicher Schritt für den Weg von der an der Hochkultur orientierten Kulturpolitik der ersten Phase zur stark kommerziell orientierten Phase drei war 
der Schritt zum Konzept der Kulturindustrien im Plural, der allerdings im deutschsprachigen Raum nicht unter dieser Terminologie und vor anderem intellektuellem Hintergrund vollzogen wurde. Es handelte sich dabei, was den Ausgangspunkt betrifft, im Wesentlichen um ein französisches Unternehmen: „From 1972 onwards, academics, that is to say mostly French sociologists such as Huet, Miège, Lacroix, Tremblay, Mattelart and more in particular Girard in the report he wrote for the Unesco in $1972[\ldots]$ launch the concept of ,cultural industries' in plural “" (Segers/Huijgh 2006: 6).

Im politischen Feld folgte dem terminologischen Wandel zu den Cultural Industries zuerst die UNESCO im Jahr 1980:

„The appearance of the ,cultural industries` as a more positive policy concern at the end of the 1970s was not some ,recognition' of the economic importance of commercial culture. Rather it was an opening up of a new kind of ,cultural political' space within what had previously been viewed by many in the policy establishment as degraded Americanized kitsch. This new cultural political space can be seen clearly in Augustin Girard's influential 1980 paper for UNESCO, written as head of research at the French Ministry of Culture (Girard 1982). Girard points to the huge commercial cultural sector and as a matter of urgency calls on the cultural policy establishment to take note." (O'Connor 2011: 27; vgl. auch Girard 1982; Hesmondalgh/Pratt 2005: 3)

Es folgten Großbritannien und Frankreich um 1982 mit der Übernahme des Begriffs im Plural. Diese Übernahme war eng mit der Einsicht verbunden, dass mit den Cultural Industries potenzielle ökonomische Wertschöpfung verbunden ist. Dem schlossen sich im nächsten Schritt US-amerikanische Arbeits- und Kultursoziolog*innen an, wie Paul M. Hirsch und Richard Peterson, politische und kulturelle Ökonom*innen wie Paul Dimaggio und Herbert Schiller sowie Nicholas Garnham in Großbritannien. Ab 1985 richteten dann auch Kulturökonom*innen ihre Aufmerksamkeit auf die Kulturindustrien im Plural. Zur argumentativen Rechtfertigung wurde hauptsächlich auf die Ausdifferenzierung der verschiedenen Felder der kulturellen Produktion verwiesen:

„The cultural sectors included in the concept of cultural industries cannot be treated as a unity; the complexity of the production process (technological mediation, concentration movements, labor division and organization), consumption patterns and esthetic form need a specific approach and analysis.“ (Segers/Huijgh 2006: 6)

Der Begriff der Creative Industries wurde in der Kulturpolitikliteratur erst in der Mitte der 1990er Jahre lanciert, obwohl der französische Kulturminister Jack Lang ihn bereits 1981 erstmals gebrauchte. Ab 1995 sind Creative Industries und zu- 
nehmend auch Creative Economy der empirischen Analyse von Segers und Huijgh (vgl. ebd., 5) zufolge dann viel gebrauchte Konzepte.

Zur politischen Instrumentalisierung des Creative-Industries-Konzeptes durch New Labour und zur gewählten neuen Rhetorik schreiben zwei britische Kritiker*innen:

„By adopting the phrase ,creative industries“ Britain’s New Labour government were doing the reverse: in other words bringing the creative arts into an economic policy agenda. New Labour purposefully adopted the term creative industries to replace, cultural industries' because it was regarded as a ,unifying' and ,democratising' notion. As a rhetorical device, it bridges the divide between ,high' and ,low' culture - between the mass market, popular cultural products of the cultural industries and the high art of the creative arts, now branded ,elitist'. It also bridges the divide between , art' and ,industry “ - between the demarcations of what is ,publicly supported" and what is ,commercial'“" (Galloway/Dunlop 2007: 20)

Die Einschätzung von Segers und Huijgh wiederum lautet: „The prefix ,creative‘ offers possibilities for policy makers to introduce economical aspects within cultural policy, while within the concept of cultural industries this is often rejected in the cultural field“" (2006: 8).

Die angesprochene Zurückweisung des ökonomistisch-instrumentalen Gebrauchs des Kreativitätsbegriffs im kulturellen Feld verdankt sich jenem durchaus nach wie vor lebendigen Anti-Ökonomismus, den Pierre Bourdieu (1993, 1996) den Feldern der kulturellen Produktion zuschreibt, sofern sie relative Autonomie gegenüber dem gesellschaftlichen Machtfeld, den Feldern von Wirtschaft, Politik und Bürokratie und deren Akteur*innen und Erwartungen erringen konnten.

Zum Gebrauch der Begriffe Creative Industries und Cultural Industries stellen die belgischen Wissenschaftlerinnen schließlich noch fest, dass ersteres Konzept von Ökonom*innen und in der Wirtschaftspolitik präferiert wird, während die Formulierung Cultural Industries sich größerer Akzeptanz in der Kulturpolitik, aber auch in einer Disziplin wie der Soziologie erfreue. Sie verweisen zudem darauf, dass Creative Industries gerne für die Imagepolitik neu etablierter Regierungen herangezogen werde, gleichgültig ob sozialdemokratisch oder konservativ. Ab 2005 löse der somit auch stark politisch motivierte Begriff der Creative Industries den der Cultural Industries zunehmend ab.

Für eine systematische Analyse der Diffusion von Wörtern steht seit einigen Jahren das im Rahmen des Culturomics-Ansatzes entwickelte Instrument der Nutzung digitalisierter Bücher in großem Umfang zur Verfügung. Googles Ngram Viewer zur Zeitreihenanalyse wurde mit den Textkorpora in mehreren Sprachen, für deren Analyse er herangezogenen werden kann, in der renommierten Wissenschaftszeitschrift Science im Jahre 2011 u.a. mit folgenden Worten vorgestellt: „We 
report the creation of a corpus of 5,195,769 digitized books containing $\sim 4 \%$ of all books ever published. [...] Usage frequency is computed by dividing the number of instances of the n-gram in a given year by the total number of words in the corpus in that year" (Michel et al. 2011: 176).

Entsprechende Analysen, die von mir auf der Grundlage von Googles Ngram Viewer und den für dieses Programm verfügbaren Korpora an digitalisierten Büchern durchgeführt wurden, belegen, dass sich das Wort ,creativity“ im anglophonen Sprachraum überhaupt erst seit den 1930er Jahren verbreitete.

Abbildung 2: Die relative Häufigkeit des Substantivs, creativity 'in englischsprachigen Büchern (US-amerikanisches und britisches Englisch) von 1900 bis 2008

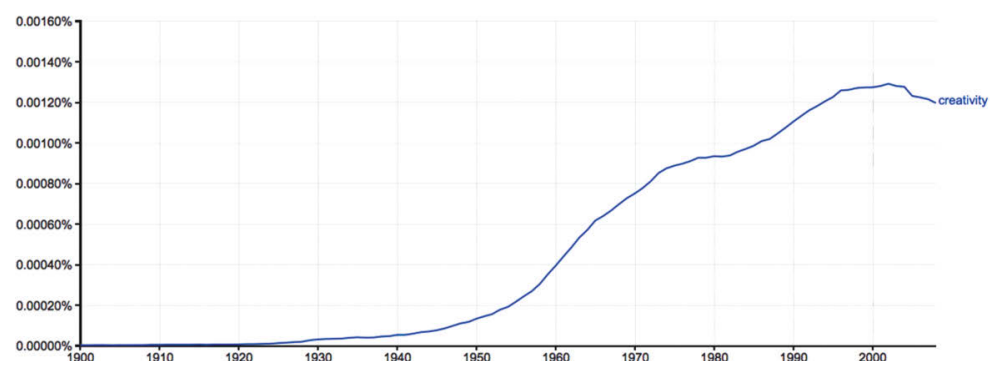

Quelle: Google Books Ngram Viewer, Kurvenglättung = 3

Es besteht weitgehend Einigkeit darüber, dass entscheidende Impulse für die Verbreitung des Kreativitätsbegriffs über verschiedene Sprachräume von jener Rede des Psychometrikers Joy P. Guilford auf der Jahrestagung der American Psychological Association 1950 ausgingen, die dieser als deren Präsident gehalten hatte. Dieser Impuls kam aus einer faktorenanalytischen, utilitaristischen Psychologie, die zwischen Kreativität und Intelligenz bzw. den neu eingeführten Konzepten „convergent thinking“ und „divergent thinking“ differenzierte. Letzteres charakterisierte er metaphorisch als, flüssig 'im Sinne von rascher Produktion einer Vielzahl von Ideen oder Lösungen für ein Problem; als ,flexibel ‘ im Sinne der Fähigkeit, eine Reihe von Problemlösungen gleichzeitig im Auge haben zu können; als ,originell' im Sinne der Schaffung von Differenz zu bekannten Lösungen und als ,elaboriert' im Sinne der Fähigkeit, die Details einer Idee zu durchdenken und umzusetzen. Zur Geschichte des Gebrauchs des Kreativitäts-Terms ist - unabhängig von der Ngram-Viewer-basierten Zeitreihe - unter dem Stichwort ,creativity“ im Jahrzehnte später erschienenen Handbuch Key Concepts in Creative Industries zu lesen: „The abstract noun ,creativity“ was first recorded in English in 1875 and was next found in the early twentieth century in phrases such as ,creative educa- 
tion' (1936), ,creative salesman' (1930) and courses in ,creative writing' (1930) (Pope 2005)“ (Hartley et al. 2012: 66).

Diese Beschreibung auf der Grundlage ausgewählter Beispiele entspricht durchaus dem leichten Anstieg der Wortverlaufskurve in den 1930er Jahren in Abbildung 2. Des Weiteren heißt es im Handbuch:

„The modern usage of creativity therefore is a product of the mid-twentieth century and the modern West. It generally implies utilitarian creativity, typified by J.P. Guilford's 1950 presidential address to the American Psychological Association in which he championed the benefits of research into creativity. Guilford went on to devise psychometric tests that were later incorporated into the well-known Torrance Tests for Creative Thinking (Sternberg and Lubart 1999).“(Ebd., 66)

,Kreativität‘ verbreitete sich als Wort im deutschsprachigen Raum wiederum erst nach dem Zweiten Weltkrieg über den ,Import‘ und die Übersetzung von ,creativity' aus den USA. Dies verdeutlichen Zeitreihenanalysen, die sich auf das deutschsprachige Korpus des Ngram Viewers stützen. Da diese Datenquelle für Zeitreihenanalysen nicht unumstritten ist, wenngleich sich die Vorbehalte in erster Linie auf historisch weiter zurückliegendes Quellenmaterial beziehen (vgl. Pechenick/Danforth/Dodds 2015; Sparavigna/Marazzato 2015), wurde von mir eine entsprechende Wortverlaufsanalyse zusätzlich mithilfe des Digitalen Wörterbuches der Deutschen Sprache (DWDS) ${ }^{4}$ erstellt. Sie bestätigte jedoch den auf Google Books basierenden Kurvenverlauf im Wesentlichen, da sie insgesamt einen deutlichen Wandel im deutschsprachigen Textmaterial ab 1955 zeigte.

4 DWDS-Wortverlaufskurve für ,Kreativität‘ erstellt durch das Digitale Wörterbuch der deutschen Sprache, https://www.dwds.de/r/plot?view=1\&corpus=dta\%2Bdwds\&norm= date $\% 2$ Bclass $\&$ smooth $=$ spline $\&$ genres $=1 \&$ grand $=1 \&$ slice $=10 \&$ prune $=0 \&$ window $=3 \mathrm{w}$ base $=0 \& \operatorname{logavg}=0 \& \operatorname{logscale}=0 \&$ xrange $=1945 \% 3 \mathrm{~A} 2000 \& \mathrm{q} 1=$ Kreativität. 
Abbildung 3: Die relative Häufigkeit des Substantivs , Kreativität ' in deutschsprachigen Büchern von 1900 bis 2008

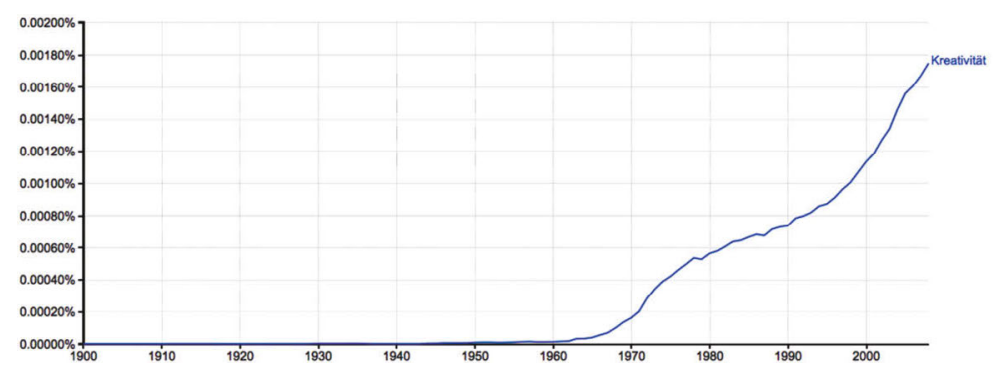

Quelle: Eigene Analyse auf der Basis von Google Books Ngram Viewer, Datensatz 2012, Kurvenglättung $=3$

Die entscheidenden Impulse kamen dabei von dem von Joy P. Guilford maßgeblich angestoßenen ,creativity movement ( vgl. Bycroft 2012). ${ }^{5}$ Das „,creativity movement" im Sinne von Michael Bycroft war von Anfang an heterogen. Psychometriker*innen wie Guilford wurden von Vertreter*innen der humanistischen Psychologie (z.B. Carl Rogers, Frank X. Barron) wie auch der Psychoanalyse (z.B. Donald Winnicot) flankiert. In Europa wurde die Idee, dass es der Gesellschaft an Kreativität mangle, nicht zuletzt von den antisystemischen Bewegungen, die sich in den 1960er Jahren formierten, aufgegriffen - so etwa von bekannten Vertreter*innen der Künstlerkritik wie Raoul Vaneigem, Mitglied der Situationistischen Internationale von 1961 bis 1970, oder von Joseph Beuys. Gemeinsam mit Klaus Staeck verfasste Beuys 1972 ein Manifest zur Gründung einer Freien Internationalen Hochschule für Kreativität und Interdisziplinäre Forschung. ${ }^{6}$ Und 1983 pflanzte er einen Baum für eine Schule der Kreativität.

Solche Initiativen beflügelten später die Vorstellung einer nicht zuletzt auf Impulse aus künstlerischen Feldern gestützten Herausbildung des ,neuen Geistes des Kapitalismus“" (Boltanski/Chiapello 2018) bzw. eines ,ästhetischen Kapitalismus“, ein von Andreas Reckwitz (2012: 11) vor dem Hintergrund der Annahme eines

5 Nicht zu verwechseln ist das „creativity movement“ im Sinne von Bycroft mit der Selbstbezeichnung einer rassistischen, suprematistischen Gruppe in den USA, die dieses Label gebraucht und zudem „Creativity“ als Namen ihrer anti-christlichen Religion wählte (vgl. Dobratz 2001).

6 Das „Manifest zur Gründung einer Freien Internationalen Hochschule für Kreativität und Interdisziplinäre Forschung" ist abrufbar unter http://4.bp.blogspot.com/-MsEeM6wkp2I/UX_6MmCG93I/AAAAAAAAAv4/xqNehnwQVI/s1600/339x472.jpg. 
maßgeblichen Einflusses des künstlerischen Feldes auf die Form des zeitgenössischen Kapitalismus vorgeschlagener Begriff. Bei den französischen Autor*innen findet sich hingegen ein Aneignungs- und Kooptationsargument in kritischer Absicht. Während die traditionelle ,Sozialkritik‘ primär auf die Lösung sozioökonomischer Probleme durch Verstaatlichung und Umverteilung zielte, kreist die von Luc Boltanski und Ève Chiapello als „Künstlerkritik“ bezeichnete Spielart der Kritik um „ein Ideal der individuellen Autonomie, der Selbstverwirklichung und der Kreativität, das im Widerspruch zu allen Formen hierarchischer Machtverhältnisse und sozialer Kontrolle steht“" (Boltanski 2007: o.S.). Dabei ist zu beachten, dass der von Pierre Bourdieu übernommene, ursprünglich noch im engen Sinne gebrauchte Begriff der Künstlerkritik von Boltanski und Chiapello weit ausgedehnt wurde, im Wesentlichen sogar in eine ähnliche Richtung wie Richard Floridas (2002) „Creative Class“, wie aus einem mit den beiden von Yann Moulier-Boutang geführten Interview hervorgeht:

„Es ist zu beachten, dass die Künstlerkritik heute vor allem von Personen getragen wird, die im oberen Teil der sozio-kulturellen Hierarchie platziert sind, die über eine Hochschulausbildung verfügen, die häufig in kreativen Sektoren arbeiten (dem Marketing, der Öffentlichkeitsarbeit, den Medien, der Mode, dem Internet etc.) oder auch in den Finanzmärkten oder in Beratungsgesellschaften [...].“ (Boltanski in Moulier-Boutang 2000: 137, Übersetzung aus dem Französischen U.W.)

Aus Sicht der Verteidiger*innen des Kapitalismus bot diese von Künstler*innen und Intellektuellen, aber auch von anderen Gruppen, getragene Form der Kritik den Vorteil, durch gewisse Umdeutungen und Glättungen mit einem liberal gemäBigten Kapitalismus durchaus vereinbar zu sein. In der Welt der Unternehmen und der Arbeit wurde diese Umdeutung - so die Kooptationsthese - größtenteils durch die Vordenker*innen neuer Formen des Managements geleistet, die eine liberalisierte Spielart des Kapitalismus propagierten, der auch die Erfahrungen der 1960er und 1970er Jahre integrieren sollte.

Während Guilford, wie oben beschrieben, schon 1950 in seiner Rede auf dem Kongress der US-amerikanischen Gesellschaft für Psychologie neben Intelligenz auch die Berücksichtigung der mit dieser empirisch nur schwach korrelierenden Kreativität in der Form des „divergent thinking“ gefordert hatte, kam das Wort ,Kreativität' in deutschsprachigen Büchern erst deutlich später auf, nämlich Mitte der 1960er Jahre. Das verweist einerseits darauf, dass es sich um einen eingedeutschten US-Import handelt und andererseits darauf, dass auch die sich entfaltende 1968er-Bewegung in diesem Zusammenhang eine nicht unwesentliche Rolle spielt.

Im Hinblick auf das Adjektiv ,kreativ', für das in der Zeitreihenanalyse seit 1800 die Verbreitung von Äquivalenten in fünf Sprachräumen berücksichtigt werden 
konnte (US-amerikanisches und britisches Englisch, Spanisch, Französisch und Deutsch), ließ sich mithilfe des Ngram Viewers erkennen, dass das Wort ,creative“ in den USA und in Großbritannien bereits im 19. Jahrhundert eine Rolle spielt. Darauffolgend zeichnet sich ein deutlicher Anstieg in der ersten Hälfte des 20. Jahrhunderts $\mathrm{ab}$, während der Ausdruck zunächst in der spanischsprachigen, dann in der frankofonen und deutschsprachigen Welt überhaupt erst in den 1960er Jahren aufkommt.

Ein entsprechender Blick in sechs Sprachräume für das Substantiv ,creativity und die Äquivalente ,Kreativität', ,créativité', ,creatividad' und ,creatività ' seit 1950 zeigt nochmals, dass es sich um die Ausbreitung eines im US-amerikanischen Kontext gebrauchten Wortes handelt. Es kommt erst in den 1960er Jahren im britisch-englischen, im spanischen, im italienischen und im deutschen Sprachraum auf.

Abbildung 4: Relative Häufigkeit der Verwendung von Wörtern in digitalisierten Büchern 1950 bis 2008, für ,,creativity “ in US-amerikanischem und britischem Englisch, für ,Kreativität“ im Deutschen, für ,,creativité “ im Französischen, für ,, creatividad“ im Spanischen und für ,,creatività “ im Italienischen

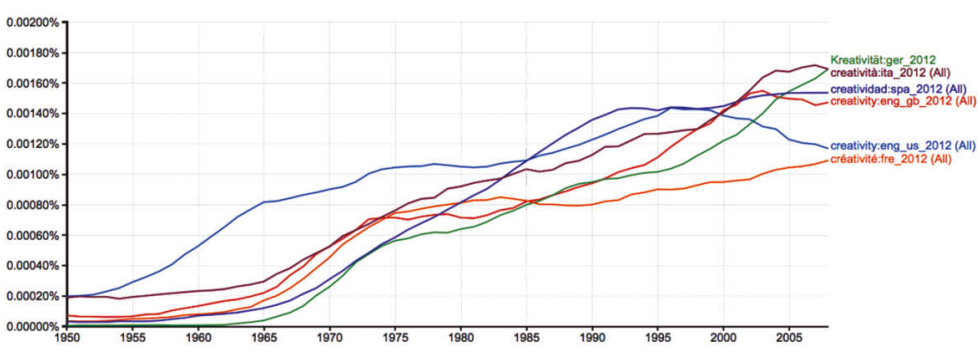

Quelle: Eigene Analyse auf der Basis von Google Books Ngram Viewer, Datensätze 2012, case-insensitive, Kurvenglättung $=3$

Bei Creative Industries und der Verbreitung dieses Ausdrucks handelt es sich hingegen nicht um einen Diffusionsprozess, der im US-amerikanischen Sprachraum seinen Ausgang nimmt, sondern um einen Export aus dem britischen Sprachraum. Dort kommt der Begriff 1995 und 1996 im Zuge des Aufstiegs von New Labour und der Cultural-Industries-Politik der partiell auf neoliberalen Kurs umgeschwenkten sozialdemokratischen Partei auf. Im Begriff der Creative Industries ist durchaus eine Art contradictio in adjecto zu sehen, wenn etwa ein Big-C-Kreativitätsbegriff zugrunde gelegt wird, dessen klassische Konzeption im Handbuch der Creative Industries niemand anderem als Immanuel Kant und dessen ,Geniebegriff‘ zugeschrieben wird: ,The pairing of ,creative‘ with ,industries“ may seem 
to be a contradiction in terms, if we adopt a time-honoured Western European perspective on creativity derived from Kant's aesthetics and represented in the ideal type of the ,creative genius “" (Hartley et al. 2012: 66). In den beiden romanischen Kontexten und im US-Sprachraum fasst der Terminus allerdings stärker Fuß als im deutschen Sprachraum. In Deutschland wird bis heute kaum von ,Kreativindustrien' gesprochen. In die administrative deutsche Sprache eingegangen ist statt ,Kreativindustrie“ oder ,Kreativindustrien“ vielmehr die aus dem Ausdruck Creative Economy abgeleitete ,Kreativwirtschaft'. Dieses Konzept gewinnt im deutschsprachigen Raum sogar größeres Gewicht als in anderen Kontexten.

Angesichts der speziellen politischen Einbettung des Aufstiegs des Kreativitätsbegriffs vor allem im Zusammenhang mit dem Begriff der Creative Industries ist es nicht erstaunlich, dass im britischen Kontext von einer „decade of creativity“ geschrieben wurde (vgl. McRobbie 2011: 32), die mit der Regierungsübernahme durch Tories und Liberale in London im Jahre 2010 endete.

Was den deutschsprachigen Raum betrifft, sind allerdings die erheblichen Divergenzen des Creative Turn - der von Ann Harris (2014) gewählte Titel einer Monografie über den kulturpolitischen Wandel - und seiner Klassifikationen, die nationalen Grenzen folgen, nicht zu übersehen. So wurde in Deutschland im Jahr 2009 von der Wirtschaftsministerkonferenz (WMK) eine neue Terminologie in Zusammenhang mit der Neufassung der Wirtschaftsstatistik nach einem einfachen dualen Schema eingeführt. Es handelt sich um die Idee einer Klassifikation eines Teils des erwerbswirtschaftlichen Sektors in Deutschland entweder als Kultur- oder aber als Kreativwirtschaft. Diese Differenzierung folgte allerdings nicht der Logik der Creative Industries, wie bereits der Verzicht auf den Begriff der Industrien zeigt. Die Definition lautet: „Unter Kultur- und Kreativwirtschaft werden diejenigen Kultur- und Kreativunternehmen erfasst, welche überwiegend erwerbswirtschaftlich orientiert sind und sich mit der Schaffung, Produktion, Verteilung und/oder medialen Verbreitung von kulturellen/kreativen Gütern und Dienstleistungen befassen“" (BMWi 2014: 13).

Die unterschiedenen zwei Branchen bzw. Teilmärkte der Kreativwirtschaft, welche als besonders stark kommerziell orientierte Sphären angesehen werden können, und die neun der Kulturwirtschaft sind hier jeweils in der Reihenfolge ihrer relativen Anteile an der gesamten Bruttowertschöpfung der Kultur- und Kreativwirtschaft im Jahr 2017 aufgelistet (vgl. BMWi 2019: 24):

A Kreativwirtschaft: 1. Software-/Games-Industrie (27,2 Prozent), 2. Werbemarkt (10,5 Prozent)

B Kulturwirtschaft: 1. Pressemarkt (11,7 Prozent), 2. Designwirtschaft (9,4 Prozent), 3. Rundfunkwirtschaft (7,8 Prozent), 4. Filmwirtschaft (7,1 Prozent), 5. Markt für darstellende Kunst (6,9 Prozent), 6. Architekturmarkt (6,2 Prozent), 
7. Musikwirtschaft (6,0 Prozent), 8. Buchmarkt (4,8 Prozent), 9. Kunstmarkt (1,3 Prozent), 10. Sonstige (1,1 Prozent).

Eine vergleichbare Trennung zwischen einer in hohem Maße über heteronome Teilmärkte definierten Kreativwirtschaft - was dem kultur- und wirtschaftspolitisch eingesetzten Kreativitätsbegriff in Deutschland eine hochgradig kommerzielle Konnotation verschaffte - und einer Wirtschaftssphäre, für welche am Kulturbegriff festgehalten wird, wurde weder in Österreich noch in der Schweiz vollzogen. In beiden Ländern ist nur noch von Kreativwirtschaft und nicht mehr von Kulturwirtschaft die Rede. Eine symbolische Barriere gegenüber hochgradig kommerziellen Sphären wurde beseitigt. Somit umfasst die Kreativwirtschaft in Österreich vor dem Hintergrund der ökonomischen und politischen Instrumentalisierung des Kreativitätsbegriffs jene ,erwerbsorientierte Unternehmen, die sich mit der Schaffung, Produktion und (medialen) Distribution von kreativen und kulturellen Gütern sowie Dienstleistungen beschäftigen“ (Kreativwirtschaft Austria und Wirtschaftskammer Österreich 2017: 15).

Die Kreativwirtschaft in Österreich erstreckt sich nach einer Reduktion der Zahl der unterschiedenen bzw. berücksichtigten Bereiche im aktuellen Kreativwirtschaftsbericht über nunmehr zehn Teilbereiche. Wie in Deutschland stellen „Software und Games“ (ebd., 15) das größte Segment dar, gemessen am Anteil der Beschäftigten sowie an Umsatz und Bruttowertschöpfung. Dieses wird als die „dynamischste Branche“ und als „Innovationstreiber“ der Kreativwirtschaft beschrieben (ebd.). In der Reihenfolge der in Klammern ausgewiesenen Anteile an der Bruttowertschöpfung ergibt sich hinsichtlich der Branchen bzw. Teilmärkte für 2014 folgendes Bild (vgl. ebd., 60): 1. Software und Games (34 Prozent), 2. Markt für darstellende Kunst (15 Prozent), 3. Werbung (14 Prozent), 4. Buch und Verlagswesen (14 Prozent), 5. Architektur (10 Prozent), 6. Filmwirtschaft (inkl. Fotografie) (5 Prozent), 7. Radio und TV (5 Prozent), 8. Design (1 Prozent), 9. Musikwirtschaft (1 Prozent) und 10. Bibliotheken und Museen, botanische und zoologische Gärten (nur privatwirtschaftlicher Sektor und keine Berücksichtigung in der Statistik).

In der Schweiz erfolgte in jüngerer Zeit eine Abkehr von dem ursprünglich verfolgten einfachen Modell, die Kreativwirtschaft ausschließlich nach einer sektoralen Logik und einer Addition von vorab als kreativ definierten Wirtschaftsbranchen abzugrenzen. Seit 2016 wird versucht, den Blick auf die ,kreative Beschäftigung' in der Gesamtwirtschaft zu richten. Weder wurde zuvor zwischen Kultur- und Kreativwirtschaft im deutschen Sinn differenziert noch erfolgte eine ausschließliche Beschränkung auf den Typus der in Deutschland und Österreich berücksichtigten Wirtschaftsbranchen. Abgezielt wird vielmehr auf eine Identifikation von ,kreativen Berufen“ in allen Branchen der Wirtschaft. Vorbild für diese 
Neuerung in den rezenten nationalen Kreativwirtschaftsberichten der Schweiz von Christoph Weckerle, Roman Page und Simon Grand (2016, 2018) sind entsprechende Analysen, die in Großbritannien in jüngerer Zeit vorgenommen wurden (vgl. Bakhshi/Freeman/Higgs 2013). Damit ist im Zusammenhang mit dem statistischen und wirtschaftspolitischen Gebrauch des Kreativitätsbegriffs erneut ein Import eines britischen Modells in ein kontinentaleuropäisches Land zu beobachten. Es könnte auch in anderen Ländern Schule machen, da dieses Modell immanent betrachtet bedeutend überzeugender ist als etwa das in Österreich zugrunde gelegte.

Der Creative-Economy-Begriff des Schweizer Modells von Weckerle, Page und Grand (2016) kennt auf allgemeiner Ebene drei Berufsgruppen: a) „Nicht-Spezialist*innen“ im Sinne von Erwerbstätigen, die in einer „kreativen Branche“ einem „nicht-kreativen Beruf“ nachgehen, wofür etwa eine in einem Verlag tätige Buchhalterin ein Beispiel wäre; b) „Spezialist*innen“ im Sinne von Erwerbstätigen, die in einer „kreativen Branche“ einem „kreativen Beruf“ nachgehen, wie beispielsweise Tänzer*innen in einem Ensemble; c) „Eingebettete“, die in einem „kreativen Beruf" in einer Branche außerhalb der Creative Industries arbeiten, exemplifiziert etwa durch eine bei einem Finanzdienstleister tätige Game-Designerin (ebd., 5ff.). Die in der Schweiz unter dem Oberbegriff der Kreativwirtschaft berücksichtigten Bereiche der Creative Industries umfassen 13 Branchen. Es sind dies zum einen die beiden Kreativwirtschaftsbranchen im deutschen Sinn, die Software- und Games-Industrie sowie der Werbemarkt. Darüber hinaus werden in dortiger Terminologie noch Musikwirtschaft, Buchmarkt, Kunstmarkt, Filmwirtschaft, Rundfunkmarkt, Markt der Darstellenden Kunst, Designwirtschaft, Architekturmarkt, Kunsthandwerk, Pressemarkt und Phonotechnischer Markt aufgelistet.

Im Zusammenhang mit einer weiteren trichotomen Differenzierung werden auch im Schweizer Kreativwirtschaftsbericht Einblicke in den zugrunde gelegten Kreativitätsbegriff gegeben. Es ist ein bestimmtes Verständnis von Kreativität und ihren Bedingungen, welches es aus der Sicht der drei Schweizer Autoren nahelegt, nicht von einer Zugehörigkeit zu ausgewählten Branchen auszugehen, sondern vielmehr ,spezifische Haltungen, Praxen und Prozesse“ (ebd., 73) und deren faktische Wirkung in Kultur, Wirtschaft und Wissenschaft zu berücksichtigen. So kennt die eingeführte Trichotomie ein Zentrum, einen ,creative core‘. Dessen Definition verweist zunächst auf einen „Kreativitätsbegriff, der in enger Verbindung steht zu künstlerischer Kreation“ (ebd.). Eine Reduktion auf künstlerische Produktion erscheint den Autoren jedoch als zu eng, denn auch „Experimente, Improvisationen, das Hacking bestehender Systeme, kritische Auseinandersetzungen usw. können je nach Kontext Ausgangspunkt kreativer Behauptungen und Prozesse sein“ (ebd.). Als soziale Bedingung der Möglichkeit dieser Art von Kreativität wird wiederum vor allem Unsicherheit angesehen: 
„Zentrales Moment dabei ist die Tatsache, dass die jeweiligen Akteurinnen und Organisationen in unsicheren Konstellationen tätig sind und sich mit der Entwicklung alternativer, d.h. nicht linear aus dem Status quo ableitbarer Szenarien beschäftigen. Die Auseinandersetzung mit alternativen Modellen und neuen Möglichkeiten findet statt und kann genauso in wissenschaftlichen Laboratorien, in Design-Agenturen, in technologischen Ventures, in der freien Theaterszene, in unternehmerischen Initiativen etablierter Grosskonzerne, in neuen Modellen journalistischer Berichterstattung wie in Kunstausstellungen wirken.“(Ebd.)

Problematisch an dieser empirischen Prämisse erscheint nicht zuletzt, dass in der Formulierung Akteur*innen in abgesicherten Konstellationen der Status potenziell kreativer Akteur*innen abgesprochen wird. Zumindest liegt eine solche Auslegung nahe. Gerade institutionelle und personelle Autonomie als Bedingung für Kreativität (vgl. Bourdieu 1993) bedarf im Falle der Wissenschaft wie auch der Kunst oftmals sozialer Absicherung. Daran erinnert in der Kunst ein Träger einer großen symbolischen Revolution wie Edouard Manet und daran erinnern zahlreiche innovative Wissenschaftler*innen, die ihre Neuerungen als Professor*innen in öffentlichen Hochschulen schufen bzw. über diese durchsetzten.

Einem als ,collocated sphere' bezeichneten zweiten Bereich werden Organisationen und Branchen zugeordnet, welche notwendige Rahmenbedingungen technologischer, infrastruktureller, finanzieller oder anderer Natur sicherstellen, die für eine wirksame Verbreitung, Umsetzung und Durchsetzung neuer Ideen, Entwürfe oder Behauptungen von Bedeutung sind. Auch diesen Akteur*innen wird im Schweizer Modell Kreativität zugeschrieben, weil sie Möglichkeitsräume stark erweitern oder auch beschränken können. In einem dritten, als „extended sphere“ bezeichneten Bereich werden schließlich Akteur*innen verortet, welche in der Lage sind, ,wichtige, eigenständige und teilweise außerordentlich kreative Austauschbeziehungen“ (Weckerle/Page/Grand 2016: 73) zwischen den beiden skizzierten Sphären über multiple Transfer- und Übersetzungsleistungen sicherzustellen.

Angesichts der Aneignung des Kreativitätsbegriffs durch die individualistische ,unternehmerische Kultur' (vgl. Heap/Douglas/Ross 1992) ist kaum überraschend, dass sich auch die Kritik dieser Appropriation und die Entfaltung der Rhetorik der Kreativität im Interesse der Ausrichtung von Kunst und Kultur an externen Zielen - von wirtschaftlichen, wie Wirtschaftswachstum und Arbeitsmarktimpulsen, bis hin zu sozialen, wie Nachhaltigkeit, Diversität und kollektiver Identität - im intellektuellen Feld im Rahmen einer Reihe von Symposien und in Form von zahlreichen Stellungnahmen und Publikationen entfaltete. Sie wurde und wird getragen insbesondere von Repräsentant*innen der „dissidenten Kultur“ im Sinne von Mary Douglas (1996) bzw. aus Kreisen jener ,Künstlerkritik‘, die den Kreativitätsbegriff in den 1960er und 1970er Jahren gegen die in den westlichen Ländern damals noch verbreitete „hierarchische Kultur“ eines wohlfahrtsstaatlich geprägten Kapitalismus 
mobilisierte. $\mathrm{Zu}$ denken wäre in diesem Zusammenhang u.a. an kritische Stellungnahmen von Bröckling $(2007,2010)$, van den Berg (2009), Galloway und Dunlop (2007), Goldsmith (2011), Jagodzinski (2011), O'Connor (2011), Peck (2005), Rosler (2010, 2011), Bruell (2013) und Wuggenig (2016, 2017) bzw. an Beiträge in Textsammlungen in von Osten (2003), Lovink und Rossiter (2007), Raunig und Wuggenig (2007, 2016), Menke und Rebentisch (2011) oder Beyes und Metelmann (2018).

Stellvertretend mögen für diese Art von Kritik der Kreativität zwei Äußerungen stehen: eine von Ulrich Bröckling, der vorschlug ,das Wort vorübergehend in den Giftschrank zu stellen“ (2010: 50); die andere von Stuart Hall, der vor dem Hintergrund der Instrumentalisierung des Wortes Kreativität seine weitere Verwendbarkeit infrage stellte: „More recently the terms creativity and innovation have been $[\ldots]$ assimilated to technological, commercial, managerial practices, in selfinflating and commodified ways which make them virtually unusable“ (2010: X).

\section{LITERATUR}

Amabile, Teresa M. (1996): Creativity in context. Update to the social psychology of creativity, Boulder, Colorado/Oxford: Westview Press.

Bakhshi, Hasan/Freeman, Alan/Higgs, Peter (2013): A Dynamic Mapping of the UK's Creative Industries, London: Nesta.

Becker, Howard S. (2017): „Creativity Is Not a Scarce Commodity“, in: American Behavioral Scientist 61, H. 12, S. 1579-1588.

van den Berg, Karen (2009): „Kreativität. Drei Absagen der Kunst an ihren erweiterten Begriff", in: Stephan A. Jansen/Eckhard Schröter/Nico Stehr (Hg.), Rationalität der Kreativität? Multidisziplinäre Beiträge zur Analyse der Produktion, Organisation und Bildung von Kreativität, Wiesbaden: Springer VS, S. 207-224.

Beuys, Joseph/Staeck, Klaus (1972): „Manifest zur Gründung einer Freien Internationalen Hochschule für Kreativität und Interdisziplinäre Forschung", http://4.bp.blogspot.com/-MsEeM6wkp2I/UX_6MmCG93I/AAAAAAAAAv4/xqNehnwQVI/s1600/339x472.jpg

Beyes, Timon/Metelmann, Jörg (Hg.) (2018): The Creativity Complex: A Companion to Contemporary Culture, Bielefeld: transcript.

Boden, Margarete (2004): The Creative Mind: Myths and Mechanisms [1990], London: Routledge.

Boltanski, Luc/Chiapello, Ève (2018): The New Spirit of Capitalism, (frz. 1999), London/New York: Verso.

Dies. (2002): „The New Spirit of Capitalism“, Paper to be presented to the Conference of Europeanists, Chicago, 14.-16. März 2002, 
http://www.darkmatterarchives.net/wp-content/uploads/2011/11/boltanskiSPIRITofCapitalism.pdf

Boltanski, Luc (2007): „Leben als Projekt. Prekarität in der schönen neuen Netzwerkwelt", in: polar 2 , http://www.polar-zeitschrift.de/polar_02.php?id=69\#69 Bourdieu, Pierre (1993): The field of cultural production. Essays on art and literature, hg. v. Randall Johnson, Cambridge: Polity.

Ders. (1996): Die Regeln der Kunst, Frankfurt a.M.: Suhrkamp.

Ders. (2013): Manet. Une révolution symbolique. Cours au Collège de France (1998-2000) suivis d'un manuscrit inachevé de Pierre et Marie-Claire Bourdieu, Paris: Seuil.

Bröckling, Ulrich (2007): Das unternehmerische Selbst, Frankfurt a.M.: Suhrkamp. Ders. (2010): ,,Kreativ? Das Wort ist vergiftet‘. Ein Gespräch mit dem Soziologen Ulrich Bröckling über Illusion und Wirklichkeit, über Utopie und Selbstausbeutung im Alltag der neuen Selbstständigen. Von Thomas Assheuer“, in: Die ZEIT vom 04.11.2010, S. 50-51.

Bruell, Cornelia (2013): Kreatives Europa 2014 - 2020. Ein neues Programm auch eine neue Kulturpolitik? Bonn: Ifa-Edition Kultur und Außenpolitik.

Bundesministerium für Wirtschaft und Energie (BMWi) (2014): Monitoring zu ausgewählten wirtschaftlichen Eckdaten der Kultur- und Kreativwirtschaft 2013, Berlin.

Ders. (2019): Monitoringbericht Kultur- und Kreativwirtschaft 2018, Langfassung, Berlin.

Burns, Tom R./Machado, Nora/Corte, Ugo (2015a): „The sociology of creativity: Part I: Theory: The social mechanisms of innovation and creative developments in selectivity environments“, in: Human Systems Management 34, S. 179-199.

Dies. (2015b): „The sociology of creativity: Part II: Applications: The socio-cultural contexts and conditions of the production of novelty", in: Human Systems Management 34, S. 263-286.

Bycroft, Michael (2012): „Psychology, Psychologists, and the Creativity Movement: The Lives of Method Inside and Outside the Cold War", in: Mark Solovey/Hamilton Cravens (Hg.), Cold War Social Science, New York: Palgrave Macmillan, S. 197-214.

„Cambridge Academic Content Dictionary: creativity“, https://dictionary.cambridge.org/dictionary/english/creativity

Caves, Richard (2000): Creative Industries: Contracts between Art and Commerce, Cambridge: Harvard University Press.

Collins, Randall (1987): „A micro-macro theory of intellectual creativity: the case of German idealist philosophy“, in: Sociological Theory 5, S. 47-69.

Cropley, Arthur (2011): „Definitions of Creativity“, in: Runco/Pritzker, Encyclopedia of Creativity, Bd. I, S. 358-369. 
Csikszentmihalyi, Mihaly (2010): Kreativität. Wie Sie das Unmögliche schaffen und Ihre Grenzen überwinden, (engl. 1996), Stuttgart: Klett-Cotta.

Ders. (2014): The Systems Model of Creativity, Dordrecht: Springer.

Department for Culture, Media and Sport (DCMS) (1998): „Creative Industries Mapping Document",

https://www.creativitycultureeducation.org/publication/creative-industriesmapping-document-1998

DiMaggio, Paul (2011): „Cultural Networks“, in: John Scott/Peter J. Carrington (Hg.), The SAGE Handbook of Social Network Analysis, Three Oaks, CA: Sage Publications, S. 286-300.

Dobratz, Betty A. (2001): „The Role of Religion in the Collective Identity of the White Racialist Movement", in: Journal for the Scientific Study of Religion 40, H. 2, S. 287-301.

Douglas, Mary (1996): Thought Styles, London: Sage.

Fauconnier, Gilles/Turner, Mark (2002): The way we think. Conceptual Blending and the Mind's Hidden Complexities, New York: Basic Books.

Florida, Richard (2002): The Rise of the Creative Class. And How It's Transforming Work, Leisure and Everyday Life, New York: Basic Books.

Galloway, Susan/Dunlop, Stewart (2007): „Deconstructing the Concept of ,Creative Industries “", in: International Journal of Cultural Policy 13, H. 1, S. 17-31.

Gardner, Howard (2011): Creating Minds. An Anatomy of Creativity [1993], New York: Basic Books.

Girard, André (1982): „Cultural industries: a handicap or a new opportunity for cultural development?"،, in UNESCO (Hg.), Cultural Industries: A Challenge for the Future of Culture, S. 24-40.

Giuffre, Katherine (2009): Collective Creativity: Art and Society in the South Pacific, Farnham/Burlington: Ashgate.

Glaveanu, John Vlad P./Hanson, Michael H./Baer, John/Barbot, Baptiste/Clapp, Edward P./Corazza, Giovanni E./Hennessey, Beth/Kaufman, James C./Lebuda, Izabela/Lubart, Todd/Montuori, Alfonso/Ness, Ingunn J./Plucker, Jonathan/Reiter-Palmon, Roni/Simonton, Dean Keith/Neves-Pereira, Monica Souza/Sternberg, Robert J. (2019): „Advancing Creativity Theory and Research: A Socio-cultural Manifesto“, in: The Journal of Creative Behavior 0, H. 0, S. 15, https://doi.org/10.1002/jocb.395.

Goldsmith, Kenneth (2011): Uncreative Writing: Managing Language in a Digital Age, New York: Columbia University Press.

Guilford, Joy P. (1950): „Creativity“, in: American Psychologist 5, S. 444-454.

Hall, Stuart (2010): „Foreword“, in: Helmut Anheier/Yudhishthir Raj Isar (Hg.), Cultural Expression, Creativity and Innovation. The Cultures and Globalization Series 3, London: Sage, S. IX-XII. 
Harris, Ann (2014): The Creative Turn: Towards a New Aesthetic Imagery, Rotterdam: Sense Publishers.

Hartley, John/Potts, Jason/Cunningham, Stuart/Flew, Terry/Keane, Michael/Banks, John (2012): Key Concepts in Creative Industries, London: Sage.

Hartley, John/Potts, Jason (2014): Cultural Science. A Natural History of Stories, Demes, Knowledge and Innovation, London: Bloomsbury.

Heap, Shaun Hargreaves/Douglas, Mary/Ross, Angus (1992): Understanding the Enterprise Culture: Themes in the Work of Mary Douglas, Edinburgh: Edinburgh University Press.

Hesmondhalgh, David/Pratt, Andy C. (2005): „Cultural industries and cultural policy“, in: International journal of cultural policy 11, H. 1, S. 1-14.

Hochgerner, Josef (2012): „New Combinations of Social Practices“, in: Hans-Werner Franz/Josef Hochgerner/Jürgen Howaldt (Hg.), Challenge Social Innovation, Berlin/Heidelberg: Springer, S. 87-104.

Howkins, John (2001): The Creative Economy: How People Make Money From Ideas, London/Harmondsworth: Penguin.

Jagodzinski, Jan (2011): „Creativity as Designer Capitalism: Deleuze-Guattarian Interventions“, in: Thomas Kerry/Janet Chan (Hg.), Handbook of Research on Creativity, Cheltenham (UK): Edward Elgar, S. 121-124.

Joas, Hans (1996): Die Kreativität des Handelns, Frankfurt a.M.: Suhrkamp.

Koestler, Arthur (1964): The Act of Creation, London: Hutchinson.

Kratke, Stefan (2011): The Creative Capital of Cities: Interactive Knowledge Creation and the Urbanization Economies of Innovation, Malden, MA: WileyBlackwell, S. 43-89.

Kreativwirtschaft Austria und Wirtschaftskammer Österreich (Hg.) (2017): Siebenter Österreichischer Kreativwirtschaftsbericht, Wien.

Kuhn, Thomas S. (1970): „The Structure of Scientific Revolutions“, in: International Encyclopedia of Unified Science 2, H. 2, S. 1-210.

Landry, Charles (2000): The Creative City: A toolkit for urban innovators, London: Earthscan Publications.

Lovink, Geert/Rossiter, Ned (Hg.) (2007): MyCreativity Reader: A Critique of Creative Industries, Amsterdam: Institute of Network Cultures.

Luhmann, Niklas (1988): „Über Kreativität“, in: Hans Ulrich Gumbrecht (Hg.), Kreativität - Ein verbrauchter Begriff?, München: Fink, S. 13-19.

McCraw, Thomas K. (2007): Prophet of Innovation: Joseph Schumpeter and Creative Destruction, Cambridge: Harvard University Press.

McRobbie, Angela (2011): ,Re-Thinking Creative Economy as Radical Social Enterprise“, in: Variant 41, S. 32-33.

Menger, Pierre Michel (2014): The Economics of Creativity. Art and Achievement under Uncertainty, Cambridge: Harvard University Press. 
Menke, Christoph/Rebentisch, Juliane (Hg.) (2011): Kreation und Depression. Freiheit im gegenwärtigen Kapitalismus, Berlin: Kadmos.

Michel, Jean-Baptiste/Shen, Yuan K./Aiden, Aviva P./Veres, Adrian/Gray, Matthew K./The Google Books Team/Pickett, Joseph P./Hoiberg,Dale/Clancy, Dan/Norvig, Peter/Orwant, Jon/Pinker, Steven/Nowak, Martin A./Lieberman Aiden, Erez (2011): „Quantitative Analysis of Culture Using Millions of Digitized Books“, in: Science 331, H. 6014, S. 176-182.

Mithen, Steven (1998): „A Creative Explosion? Theory of Mind, Language, and the Disembodied Mind of the Upper Paleolithic“, in: Ders. (Hg.), Creativity in Human Evolution and Prehistory, London/New York: Routledge, S. 165-191.

Moulier-Boutang, Yann (2000): „Vers Un Renouveau De La Critique Sociale“ (Interview mit Luc Boltanski und Ève Chiapello), in: Multitudes 3, S. 129142.

O'Connor, Justin (2011): „The Cultural and Creative Industries: A Critical History“, in: EKONOMIAZ. Revista vasca de Economía 78, H. 3, S. 24-47.

von Osten, Marion (Hg.) (2003): Norm der Abweichung, Wien: Springer.

Pechenick, Eitan A./Danforth, Christopher M./Dodds, Peter S. (2015): „Characterizing the Google Books Corpus: Strong Limits to Inferences of Socio-Cultural and Linguistic Evolution“, in: PLoS ONE 10, H. 10, https://doi.org/10.1371/journal.pone.0137041.

Peck, Jamie (2005): „Struggling with the creative class“, in: International Journal of Urban and Regional Research 29, H. 4, S. 740-770.

Popitz, Heinrich (2000): Wege der Kreativität, Tübingen: Mohr Siebeck.

Raunig, Gerald/Wuggenig, Ulf (Hg.) (2007): Kritik der Kreativität, Wien: Turia + Kant.

Dies. (Hg.) (2016): Kritik der Kreativität, Wien u.a.: transversal texte, https://transversal.at/books/kritikderkreativitaet

Reckwitz, Andreas (2012): Die Erfindung der Kreativität. Zum Prozess gesellschaftlicher Ästhetisierung, Berlin: Suhrkamp.

Ders. (2016): „Das Kreativitätsdispositiv und die sozialen Regime des Neuen“, in: Werner Rammert et al. (Hg.), Innovationsgesellschaft heute, Wiesbaden: Springer VS, S. 133-153.

Reinert, Hugo/Reinert, Erik S. (2006): „Creative Destruction in Economics: Nietzsche, Sombart, Schumpeter“, in: Jürgen Backhaus/Wolfgang Drechsler (Hg.), Friedrich Nietzsche 1844-2000: Economy and Society, New York: Springer, S. 55-85.

Rosler, Martha (2010, 2011): „Culture Class: Art, Creativity, Urbanism“, Part I: $e$ flux Journal 21, 12/2010, Part II: e-flux Journal 23, 03/2011, Part III: e-flux Journal 25, 05/2011, https:/www.e-flux.com/journals/ 
Rowe, David/Noble, Greg/Bennett, Tony/Kelly, Michelle (2016): „Transforming cultures? From creative nation to creative Australia“, in: Media International Australia 158, H. 1, S. 6-16.

Runco, Mark A. (1994): „Creative and imaginative thinking“, in: Vilayanur Subramanian Ramachandran (Hg.), Encyclopedia of human behavior 2, San Diego u.a.: Academic Press, S. 11-16.

Ders. (2004): „Creativity“, in: Annual Review of Psychology 55, S. 657-87.

Runco, Mark A./Pritzker, Steven R. (Hg.) (2011): Encyclopedia of Creativity, TwoVolume Set, Amsterdam u.a.: Elsevier und Academic Press.

Sarsani, Mahender Reddy (2011): „Computers and Creativity“, in: Runco/Pritzker (Hg.), Encyclopedia of Creativity, S. 231-240.

Sawyer, Keith (2011): Explaining Creativity: The Science of Human Innovation, Oxford: Oxford University Press.

Schumpeter, Joseph (1950): Kapitalismus, Sozialismus und Demokratie, (engl. 1944), Bern: Francke.

Ders. (2003): Capitalism, Socialism and Democracy [1944], London/New York: Taylor \& Francis.

Ders. (1947): „The Creative Response in Economic History“, The Journal of Economic History 7, H. 2, S. 149-159.

Segers, Katia/Huijgh, Ellen (2006): Clarifying the complexity and ambivalence of the cultural industries, Gent: Re-Creative Flanders.

Simonton, Dean K. (2013): ,What is a creative idea? Little-c versus Big-C creativity“, in: Kerry Thomas/Janet Chan (Hg.), Handbook of Research on Creativity, Cheltenham (UK)/Northhampton, MA: Edward Elgar, S. 69-84.

Sombart, Werner (2013): Krieg und Kapitalismus [1913], Barsinghausen: Unikum. Soyinka, Wole (1986): „Banquet Speech“, The Nobel Prize in Literature, Stockholm, http://old.nobelprize.org/nobel_prizes/literature/laureates/1986/soyinkaspeech.html

Sparavigna, Amelia Carolina/Marazzato, Roberto (2015): „Using Google Ngram Viewer for Scientific Referencing and History of Science“, in: Computer Science, Digital Libraries, https://arxiv.org/pdf/1512.01364.pdf

Sternberg, Robert/Lubart, Todd I. (1999): „The concept of creativity: Prospects and paradigms“, in: Robert Sternberg (Hg.), Handbook of creativity, Cambridge, UK: Cambridge University Press, S. 3-15.

Tafel-Viia, Külliki/Viia, Andres/Terk, Erik/Lassur, Silja (2014): „Urban Policies for the Creative Industries: A European Comparison“, in: European Planning Studies 4, S. 796-815.

Tafel-Viia, Külliki/Lassur, Silja (2015): Creative industries from different viewpoints. Theoretical developments, support policies, sectoral practices, Tallin: Tallinn University, School of Governance, Law and Society. 
Weckerle, Christoph/Page, Roman/Grand, Simon (2016): Kreativwirtschaftsbericht Schweiz 2016, 2nd Swiss Creative Industries Report, Creative Economies, Zürich. Dies. (2018): Creative Economy Switzerland. Creative Economy Report 2018, Zürich,

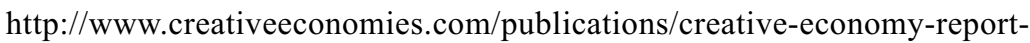
2018/creative-economy-switzerland/

Williams, Raymond (1989): „Culture is ordinary“ [1958], in: Ders., Resources of Hope, Culture, Democracy, Socialism, London: Verso, S. 3-14.

Wuggenig, Ulf (2011): „Kunst-Kunst, Street Art und ,Kreativität‘. Annäherungen mit Hilfe von Feld- und Systemtheorie“, in: Daniel Suber/Hilmar Schäfer/Sophia Prinz (Hg.), Pierre Bourdieu und die Kulturwissenschaften, Konstanz: UVK, S. 217-251.

Ders. (2016): „Kreativitätsbegriffe. Von der Kritik zu Assimilation, Vergiftung, Ausschlag. Vorwort zur Neuauflage von Kritik der Kreativität“, in: Gerald Raunig/ Ulf Wuggenig (Hg.), Kritik der Kreativität, Wien u.a.: transversal texts, S. 11-69. Ders. (2017): „Heute - 1968 - 1950. Über die Höhen und Tiefen des Kreativitätsbegriffs", in: Kunstforum International Bd. 250, Schwerpunktheft Ressource Kreativität, hg. v. Paolo Bianchi, S. 102-115. 
